\title{
EFECTOS DEL CAMBIO CLIMÁTICO EN LA DIVERSIDAD VEGETAL DEL CORREDOR DE CONSERVACIÓN COMUNITARIA RESERVA ECOLÓGICA EL ÁNGEL- BOSQUE PROTECTOR GOLONDRINAS EN EL NORTE DEL ECUADOR
}

\section{THE EFFECTS OF CLIMATE CHANGE ON VEGETATIVE DIVERSITY IN THE EL ANGEL ECOLOGICAL RESERVE-GOLONDRINAS PROTECTED FOREST COMMUNITY CONSERVATION CORRIDOR IN NORTHERN ECUADOR}

\author{
Tania Delgado ${ }^{1}$ y David Suárez-Duque ${ }^{2}$
}

\begin{abstract}
Resumen
Para estimar el impacto del cambio climático en el corredor de conservación comunitaria Reserva Ecológica El Ángel - Bosque Protector Golondrinas en el norte del Ecuador se utilizó modelos de distribución potencial generados con MARS de 413 especies vegetales, usando 19 variables bioclimáticas de Worldclim con el clima actual y para el 2080 estas mismas variables fueron estimadas de acuerdo al modelo HadCM3-A2. En base a esta información se generaron mapas de riqueza tanto para el presente como para el 2080. Para el área del corredor se han identificado zonas donde se estima que haya cambios de riqueza y donde potencialmente las especies podrían colonizar nuevas áreas o donde se extinguirán. Esto permite determinar el grado de perturbación que sufrirán los ecosistemas por efecto del cambio climático. La tendencia general de las especies estudiadas muestra que colonizarán altitudes más elevadas, cambiando la estructura de los ecosistemas naturales actuales. Toda esta información muestra que para afrontar potenciales impactos en la flora de los ecosistemas de montaña es necesario formar corredores que conecten altitudinalmente áreas naturales protegidas, que permitan la migración de especies y por ende la conservación de la biodiversidad.
\end{abstract}

Palabras clave: corredores, modelos de distribución potencial, cambio climático, tasa de recambio de especies

\begin{abstract}
In order to estimate the impact of climate change on the El Angel Ecological Reserve Golondrinas Protected Forest community conservation corridor, potential distribution models generated using MARS of 413 vegetative species were applied using 19 Worldclim bioclimatic variables for current climate, and for the year 2080, these same variables were estimated according to the HadCM3-A2 general circulation model. Based on this information, richness maps were developed for the present and for 2080. In the area of the corridor, zones were identified where changes in richness were predicted, where species could potentially colonize, or where species could become extinct. This allows the determination of the level of disturbance that the ecosystems will suffer with climate change. The general tendency of the species studied shows that they will colonize higher altitudes thus changing the structures of current natural ecosystems. All of this information demonstrates that to deal with potential impacts on the flora of mountain ecosystems it will be necessary to create corridors that will connect protected areas located at differing altitudes and thus permit species migration that will in turn conserve biodiversity.
\end{abstract}

Key words: corridors, potential distribution models, climate change, species turnover

\section{Introducción.}

Los rápidos cambios climáticos que se han registrado durante los últimos 30 años a nivel mundial han provocado numerosas transformaciones en la distribución y abundancia de las especies, causando ya alteraciones en gran variedad de ecosistemas (Chapin et al., 2000; Walther et al., 2002; Parmesan \& Yohe, 2003; Root et al., 2003). Según Thomas et al. (2004) un cambio climático mínimo produciría la extinción del $18 \%$ de las especies conocidas, mientras que un cambio máximo del $35 \%$ de especies. De acuerdo al
IV Informe del IPCC, aproximadamente el 20 - 30\% de las especies de plantas y animales posiblemente se encontraran en un aumento de peligro de extinción si la temperatura global media se excede en $1.5-2.5^{\circ} \mathrm{C}$ (IPCC, 2007). Estas proyecciones son preocupantes especialmente en áreas que se caracterizan por poseer una alta riqueza de biodiversidad, con alto índice poblacional y elevadas tasas de destrucción de hábitats naturales como es el caso de los Andes Tropicales.

Para anticiparse a estas amenazas y priorizar acciones de conservación, en los últimos años se han 
desarrollado varias herramientas de modelización para predecir la distribución y diversidad de especies. Entre estas se encuentran los modelos de distribución de especies (modelamiento de nicho ecológico) para valorar la potencial respuesta individual al cambio climático. El objetivo general de estos modelos es encontrar una relación entre factores ambientales y la presencia o ausencia de especies en un área geográfica. El modelamiento ecológico son técnicas estadísticas relativamente nuevas muy útiles en estudios de ecología, evolución, biogeografía, conservación y cambio climático (Araújo \& Williams, 2000; Graham et al., 2004; Guisan \& Thuiller, 2005; Guisan et al., 2006; Martínez-Meyer, 2005; Thuiller et al., 2005b; Elith et al., 2006; Hijmans \& Graham, 2006; Pearson, 2006; Thuiller et al., 2008).

En los modelos propuestos de escenarios de cambio global para el Ecuador se estima que el cambio climático podría causar importantes modificaciones a los actuales ecosistemas (CuestaCamacho et al., 2006; Delgado, 2008). Por ejemplo las especies vegetales van aumentar o disminuir su área de distribución potencial en distinta proporción, provocando diferentes respuestas como: desplazamientos, adaptación y/o extinción (Delgado, 2008).

El Corredor de Conservación Comunitaria Reserva Ecológica El Ángel-Bosque Protector Golondrinas es una estrategia de manejo, que promueve la conectividad entre dos áreas protegidas en el norte de Ecuador. Este corredor conecta varios ecosistemas andinos como: páramos y bosques montanos en un rango altitudinal de 900 a 4720 msnm. Esta zona une dos hotspots, el Andes Tropicales y el Túmbez-Chocó-Magdalena (Myers et al., 2000). Por esta razón la presente investigación trata de analizar el efecto del cambio climático en la diversidad vegetal de este corredor, bajo un escenario de cambio global.

\section{Materiales y métodos.}

Área de estudio.

El área establecida como Corredor de Conservación Comunitaria Reserva Ecológica El Ángel-Bosque Protector Golondrinas se encuentra dentro de las jurisdicciones de los cantones Mira, Espejo, Tulcán, y Montufar de la provincia del Carchi en el norte del Ecuador. Las principales formaciones vegetales que cubren esta zona, según Valencia et al. (1999), son: bosque siempre verde montano alto (Ceja Andina), b. de neblina montano, b. siempre verde montano bajo, b. altimontano norandino de Polylepis, matorral seco montano y páramo de frailejones; en un rango altitudinal de 900 a $4720 \mathrm{msnm}$.

Análisis de escenarios de cambio climático.

El presente análisis de escenarios se basó en un trabajo realizado por Delgado (2008) para el Ecuador continental donde analiza la evolución de la diversidad vegetal del país en un escenario de cambio global estimado para el año 2080. Para realizar un análisis a nivel del corredor se utilizó la cartografía base y de cobertura vegetal a escala 1:50.000 generada por el Laboratorio de SIG de la Corporación Grupo Randi Randi.

En este estudio se utilizó el modelo de circulación general (GCM) HadCM3, un modelo acoplado atmósfera-océano desarrollado por el Hadley Centre for Climate Prediction and Research (http://www.metoffice.gov.uk/research/hadleycentre).

El escenario elegido para este estudio fue el A2, sus características son: mayores emisiones de gases de efecto invernadero, describe un mundo muy heterogéneo caracterizado por la autosuficiencia, la conservación de las identidades locales y una población mundial en continuo crecimiento. El desarrollo económico está orientado básicamente de forma regional y los cambios tecnológicos están más fragmentados (IPCC, 2000, 2007; Arnell et al., 2004). Según Hulme \& Sheard (1999) las predicciones para el año 2080 bajo el escenario de emisiones A2, el Ecuador continental podría experimentar un aumento en la precipitación anual del $20 \%$ y un aumento de la temperatura media anual de $4.3{ }^{\circ} \mathrm{C}$.

Para generar los modelos de distribución potencial se utilizaron dos tipos de variables: variables independientes (datos climáticos actuales y futuros) y variables dependientes (registros de herbario) y el algoritmo MARS (Multivariate Adaptive Regression Splines). El algoritmo MARS, es un método estadístico que ha demostrado ser fiable en modelamiento ecológico (Moisen \& Frescino, 2002; Muñoz \& Felicísimo, 2004; Leathwick et al., 2006; Elith \& Leathwick, 2007; Delgado, 2008) el mismo que intenta explicar los patrones de presencia/ausencia actual de la especie en función de un conjunto de variables independientes construyendo valores de idoneidad para cada punto del terreno.

Para generar los datos climáticos actuales se utilizaron las 19 variables bioclimáticas de Worldclim (www.worldclim.org). Estas variables se han generado a partir de los datos de precipitación, temperatura mínima, media y máxima registrados en las estaciones meteorológicas con datos disponibles y el modelo digital de elevaciones (MDE) de 90 metros de resolución espacial derivado de la misión SRTM (Shuttle Radar Topography Mission). Todos los datos tienen formato ráster con una resolución espacial de 1 kilómetro ya que la elevación fue generalizada para adaptarla a las dimensiones de los datos de clima. Los datos utilizados se describen con mayor detalle en Hijmans et al. (2005). Las capas ráster de las variables climáticas fueron muestreadas con los temas de puntos de cada especie para obtener el fichero de muestreo correspondiente. Las grids de clima para el año 2080 fueron generadas a partir de las 19 variables bioclimáticas actuales de Worldclim 
(www.worldclim.org) modificadas de acuerdo con las previsiones derivadas del escenario HadCM3-A2. Las previsiones se proporcionan en el Tyndall Center como tablas de texto que muestran las variaciones sobre los datos climáticos del periodo 1961 - 1990 . Mediante una rutina AML (Arc Macro Language) de ArcInfo Workstation se calcularon los valores mensuales de temperatura máxima, mínima y media y de precipitación media a partir de las variaciones estimadas para el año 2080 con respecto a los valores medios medidos en el periodo 1961 - 1990. Las nuevas cuatro variables, se utilizaron para generar los valores futuros de las 19 variables bioclimáticas mediante una AML provisto por Worldclim.

Los registros de las colecciones botánicas se obtuvieron de la base datos TROPICOS del Missouri Botanical Garden. Del total de especies disponible en la base de datos TROPICOS (MO) se seleccionó aquellas que tenían $\geq 15$ presencias únicas por especie. En concreto se seleccionaron cinco familias de plantas vasculares (Bignoniaceae, Bromeliaceae, Gesneriaceae, Lauraceae y Leguminosae), algunas especies de páramo pertenecientes a varias familias (Poaceae, Cunoniaceae, Juncaceae, Loranthaceae, Caryophyllaceae, Ranunculaceae, Polygalaceae, Asteraceae, Rosaceae, Pteridaceae, Lycopodiaceae y Polypodiaceae), y el género Anthurium Schott (Araceae). En total se modelizaron 413 especies (17 716 ejemplares de herbario). Estas especies fueron elegidas por no presentar problemas en su revisión taxonómica y porque representan una gran variedad de hábitats.

Con los registros de las especies seleccionadas en formato ráster con tamaño de píxel de 1 kilómetro se construyeron modelos de idoneidad. Para la construcción de estos modelos se empleo una técnica estadística multivariante MARS (Multivariate Adaptive Regression Splines), desarrollado por Friedman (1991) que se basa en la construcción de modelos flexibles ajustando los datos a regresiones lineales por segmentos, donde la pendiente de la ecuación cambia de un intervalo a otro. Para generar estos modelos se empleó MARS 2.0 (www.salfordsystems.com). Mediante rutinas de este software, se realizó un total de 30 modelos diferentes para cada una de las especies, variando la complejidad de los modelos generados. Posteriormente se seleccionó el modelo que presentaba mejor bondad de ajuste (mayor valor de AUC).

Para el control del ajuste de predicción de los modelos generados, se utilizó el estadístico AUC (Area Under the Curve) estimado a partir de la curva ROC (Receiver Operating Characteristics) (Hanley \& McNeil, 1982). La curva ROC es la representación gráfica de la capacidad discriminativa de un determinado modelo a partir de todos sus puntos de corte posibles. Para cada punto de corte se elaboró una tabla umbral o matriz de confusión con todos los valores de sensibilidad (casos positivos) y especificidad (casos negativos). El valor de AUC está entre 0.5 y 1 (Fielding \& Bell, 1997), un valor de 0.5 equivale a una clasificación al azar, mientras que un valor de 1 indica un ajuste perfecto, es decir, que todos los casos han sido correctamente clasificados. AUC 0.9 - 1, excelente; 0.8 - 0.90, bueno; 0.7 - 0.8, razonable; 0.6 - 0.7, pobre; 0.5 - 0.6, falla (Swets, 1988). En el caso específico de este estudio (413 especies) el valor medio del AUC es de 0.98 (excelente). Los modelos generados se muestrearon en ArcInfo y se calcularon la curva ROC y el AUC con SPSS 12.0. Una vez obtenido el mejor modelo con el clima actual, este se proyectó con el clima futuro. Los modelos ráster (grids) de idoneidad actuales y futuros de cada especie fueron reclasificados a modelos de presencia/ausencia a partir del punto de corte correspondiente. De acuerdo con Liu et al. (2005), el punto de corte óptimo para MARS fue el que presentó en la curva ROC la distancia de Manhattan más corta a la esquina superior izquierda (0.1). Estos modelos se combinaron en una única grid con dos campos que contenían la información de presencia/ausencia para la actualidad y para el 2080, respectivamente. Posteriormente se añadió otro campo en el que para cada píxel se codificaba si la especie desaparecía, aparecía o se mantenía estable en el año 2080 con respecto al tiempo presente. En SPSS 15.0 se calcularon finalmente el número de píxeles ganados, perdidos y estables en relación con el área potencial inicial. Para determinar la variación de la riqueza de acuerdo a la altitud, se considero los desplazamientos altitudinales de las especies los que se determinaron usando el número de píxeles de presencia potencial de cada especie en función de la altitud.

La capacidad de migración de cada especie fue analizada con dos proyecciones, una asumiendo dispersión ilimitada y otra asumiendo dispersión nula. Para el supuesto de dispersión ilimitada se tomó en cuenta el área ganada más la que se ha mantenido estable con respecto a la de ocupación inicial. Para el supuesto de dispersión nula sólo se consideró el área que se ha mantenido estable desde la actualidad. El área ganada es la que aparece como idónea en el futuro pero no en la actualidad, el área perdida es la que aparece como idónea en el presente pero no en el futuro, el área estable se define como la que se mantiene idónea tanto en el presente como en el futuro. El porcentaje de hábitat idóneo ganado o perdido en el futuro con respecto al área potencial actual, permite determinar el grado de amenaza de las especies según su habilidad de dispersión.

Para evaluar la tasa de recambio para una zona determinada a partir de los modelos de muchas especies, fue necesario calcular para cada píxel el número de especies predichas, tanto para las condiciones climáticas actuales como futuras. Con estos valores se calculó el número de especies 
perdidas y ganadas por píxel. La diferencia permite cuantificar la intensidad de recambio de especies o diversidad beta temporal por píxel. Para el supuesto de dispersión ilimitada, la tasa de recambio se calculó tomando en cuenta la riqueza inicial y el número de especies ganadas y perdidas por píxel (Peterson et al., 2002; Thuiller, 2004; Thuiller et al., 2005a). En el caso de la dispersión nula sólo se tomó en cuenta la riqueza inicial y las especies perdidas (Thuiller, 2004). En ambos casos, una tasa de recambio de 0 indica que el conjunto de especies de un píxel no variará con el tiempo, mientras que un valor de 100 indica que todas y cada una de las especies de ese píxel serán diferentes en el futuro.

\section{Resultados y discusión.}

Cambios en la diversidad potencial de plantas: patrones de riqueza.

La riqueza potencial actual en el Corredor de Conservación Comunitaria Reserva Ecológica El Ángel-Bosque Protector Golondrinas tendría como mientras que bajo el supuesto de dispersión nula, de 61 y 186 especies por píxel (Figura $1 \mathrm{c}$ ).

En el futuro bajo el supuesto de dispersión ilimitada la riqueza potencial podría disminuir o incrementarse en algunas zonas, las áreas que podrían disminuir su riqueza están en altitudes comprendidas entre 1500 - 2000 msnm (Figura 2), el ecosistemas más afectado seria el bosque andino. Aunque en mayor proporción también podría existir pérdida de riqueza en la parte oriental del área del corredor entre 3500 y $~ 5000$ msnm (Figura 2) en zonas cubiertas por: páramo frailejones y p. de almohadillas, localizadas en la parte sur de la Reserva Ecológica El Ángel (Figura $1 \mathrm{~b})$. Bajo este mismo supuesto de dispersión, también habrá zonas que incrementen su riqueza, en altitudes comprendidas entre 1000 a $1500 \mathrm{msnm}$ y de 2000 a $3500 \mathrm{msnm}$ (Figura 2), fuera de las áreas protegidas, en el sector de La Concepción del cantón Mira, en áreas que actualmente tienen suelo desnudo, áreas intervenidas y zonas cubiertas por matorral seco (Figura 1 b).
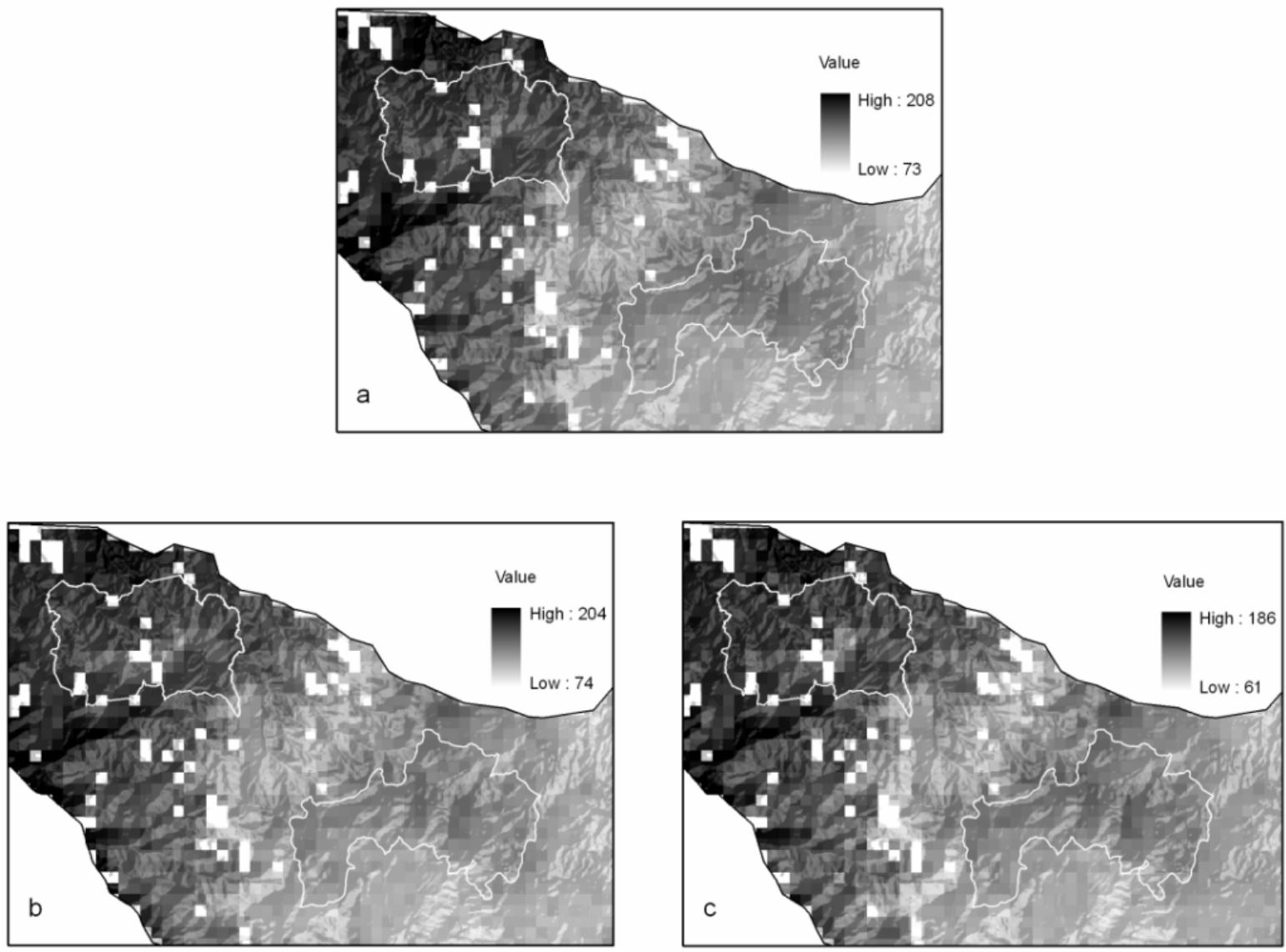

Figura 1. Variación de la riqueza potencial en el tiempo. a. Riqueza potencial actual, b. Riqueza potencial 2080 (dispersión ilimitada), c. Riqueza potencial 2080 (dispersión nula)

mínimo 73 y como máximo 208 especies por píxel (Figura 1 a). En el futuro, bajo la asunción de dispersión ilimitada, el número mínimo es de 74 y el máximo de 204 especies por píxel (Figura 1 b);
En cambio bajo el supuesto de dispersión nula podría existir una gran disminución de la riqueza, con los mayores porcentajes en altitudes comprendidas entre 1000 y 3000 msnm, y en áreas que están a más 


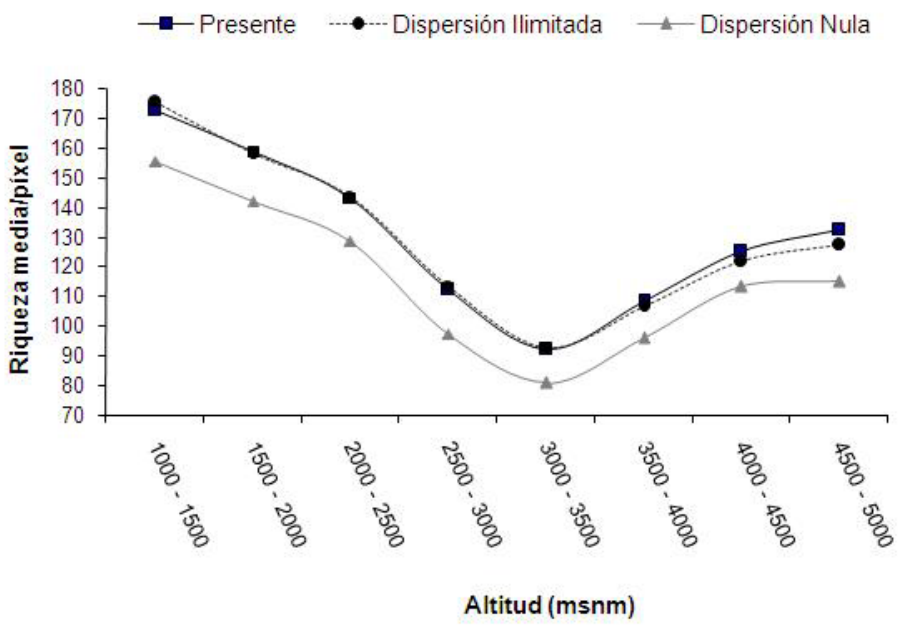

Figura 2. Patrón de riqueza potencial. Riqueza media por píxel cada $500 \mathrm{~m}$ de altitud, presente y futuro (2080) bajo el supuesto de dispersión ilimitada y dispersión nula.

de 4500 msnm (Figura 2), zonas que en la actualidad están cubiertas principalmente por bosque siempreverde montano alto, bosque andino y áreas intervenidas, que están entre Reserva Ecológica El Ángel y el Bosque Protector Golondrinas (Figura $1 \mathrm{c}$ ). Sin embargo hay que mencionar que en el futuro, pese a la posible pérdida de especies bajo los dos supuestos de dispersión, se seguirá manteniendo la alta riqueza de la parte occidental de la zona de estudio con respecto a la riqueza potencial actual (Figura 1).

Al comparar la riqueza potencial actual y futura bajo los dos supuestos de dispersión, se puede observar que a pesar de la disminución de riqueza futura que experimentará el área de estudio, si conservará el mismo patrón de riqueza (Figura 2). En la actualidad, las áreas con la más alta riqueza potencial de especies de plantas están en altitudes entre 1000 y 2500 msnm (Figura 2), zonas cubiertas por bosque andino, dentro del Bosque Protector Golondrinas y su zona de amortiguamiento (Figura 1 a). También existen zonas con alta riqueza en altitudes superiores a los 4000 msnm (Figura 2), localizadas en la Reserva Ecológica El Ángel en áreas cubiertas por diferentes tipos de páramo. De igual forma se destaca una alta riqueza en la zona que conecta estas dos áreas protegidas que es el corredor de conservación (zonas cubiertas por bosque andino, bosque siempreverde montano alto y áreas de páramo) (Figura 1 a).

Desplazamientos altitudinales.

Bajo el supuesto de dispersión ilimitada se estima que las especies en respuesta a los cambios climáticos futuros responden de forma particular y sus respuestas afectan a su vez al resto de los componentes del ecosistema, siendo capaces de migrar a distintos sitios a lo largo de la gradiente altitudinal, dando lugar a notables cambios en la estructura y composición de las comunidades, lo que ocasionara en el futuro la formación de "nuevos ecosistemas". De acuerdo a los resultados existe un desplazamiento de las especies a mayores altitudes. Esto se demuestra con algunos ejemplos de especies características de la zona y las principales respuestas de las especies al futuro cambio climático (colonización, extinción y adaptación).

- Alloplectus teuscheri (Raymond) Wiehler, arbusto escandente que se lo encuentra actualmente entre 1000 $3500 \mathrm{msnm}$, y en el futuro podría mantener estable su riqueza entre 1000 - $3000 \mathrm{msnm}$, pero sufrir pérdidas a los 3000 - $3500 \mathrm{msnm}$, e incrementar su área de ocupación en altitudes comprendidas entre $3500-4500$ msnm.

- Luzula racemosa Desv., hierba que crece por encima de $2500 \mathrm{msnm}$, en el futuro podría registrar pérdidas entre los $2500-3500$ msnm y mantener su riqueza estable por encima de los $3500 \mathrm{msnm}$ con respecto de la riqueza potencial actual.

- Cremosperma hirsutissimum Benth., hierba que se la puede encontrar entre $1000-4000 \mathrm{msnm}$, y en el futuro se estima que disminuya su área de ocupación sobre los $3000 \mathrm{msnm}$.

- Tillandsia truncata L.B. Sm., terrestre o epífita que se la puede encontrar entre 1000 - 4000 msnm, pero en el futuro podría sufrir pérdidas entre los 2500 - 3500 msnm e incrementar su área de ocupación sobre los $4000 \mathrm{msnm}$.

- Kohleria inaequalis (Benth.) Wiehler, hierba que se la puede encontrar entre 1000 - $3000 \mathrm{msnm}$, pero la proyección futura estima que podría sufrir pérdidas entre 1500 - $3000 \mathrm{msnm}$, e incrementar su presencia por encima de los $3000 \mathrm{msnm}$.

- Gynoxys buxifolia (Kunth) Cass., arbusto o arbolito que se lo puede encontrar sobre los 2500 $\mathrm{msnm}$, pero en el futuro se extinguirá entre 2500 - 3000 msnm, y ocurrirá disminución de esta especie entre 3000 - 4000 msnm, manteniéndose estable sobre los $4000 \mathrm{msnm}$.

Patrones espaciales de cambio.

Además del conocimiento de la distribución futura de las especies, es fundamental conocer y cuantificar donde las especies podrán potencialmente ganar nuevas áreas y en donde se extinguirán dando como resultado la tasa de recambio temporal de las especies (species turnover). Para el año 2080, el modelo predice que cada píxel podría ganar como máximo 70 nuevas especies (Figura 3 a), representando una ganancia relativa del 34\% con respecto a la riqueza potencial actual (Figura 1 a). Las 
mayores ganancias se darían entre 1000 - 1500 msnm, 2500 - $3000 \mathrm{msnm}$, y sobre los $4500 \mathrm{msnm}$ de altitud (Figura 4 a), en zonas que están fuera de las áreas protegidas, en áreas que actualmente están cubiertas por: bosque andino, bosque siempreverde montano alto, matorral seco, áreas intervenidas y suelo desnudo. El Bosque Protector Golondrinas tiene una mayor ganancia de especies en zonas cubiertas por bosque andino, pero también existirán ganancias aunque en menor escala en la Reserva Ecológica El Ángel en zonas que actualmente están cubiertas por: páramo de frailejones seguido de páramo de almohadillas, páramo lacustre, áreas intervenidas (Figura 3 a).
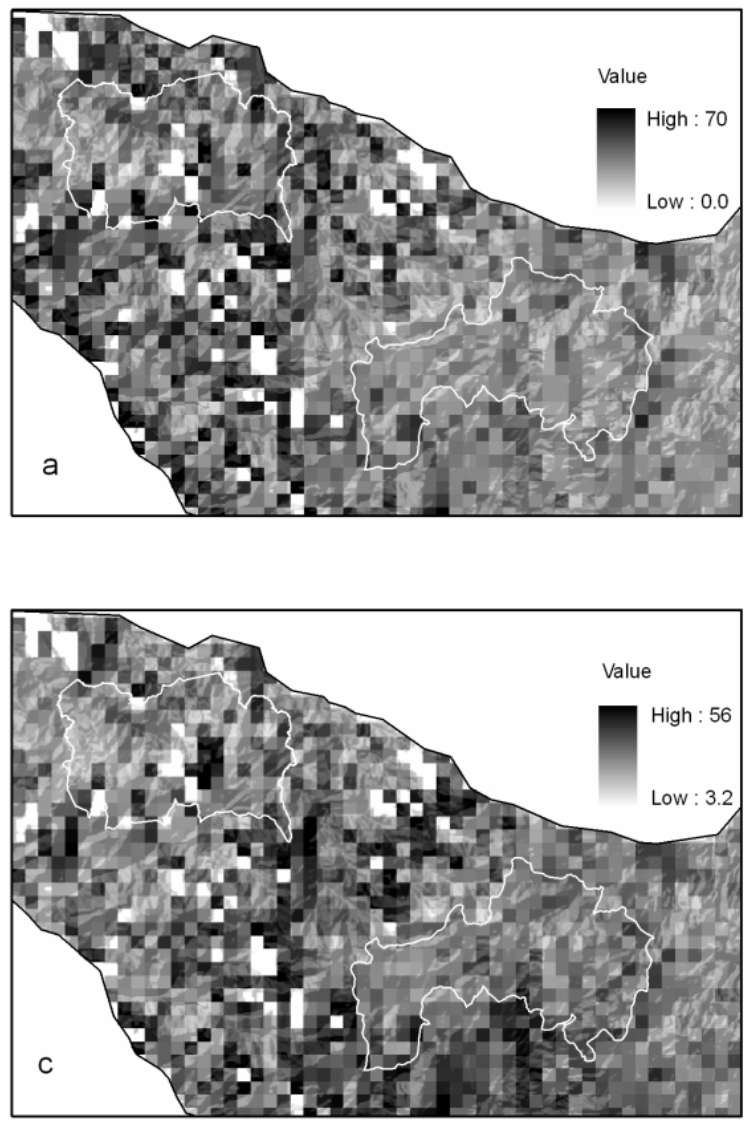

Figura 3. Patrones espaciales de cambio (HadCM3-A2, 2080). a. Ganancia, b. Pérdida, c. Porcentaje de recambio (Species turnover) bajo el supuesto de dispersión ilimitada y d. Porcentaje de recambio (Species turnover) bajo el supuesto de dispersión nula

Con respecto a las pérdidas, el modelo predice que cada píxel podría perder entre 1 y 55 especies (Figura 3 b). Tomando el escenario más pesimista (A2), la pérdida relativa sería del $26 \%$ de la riqueza potencial actual (Figura 1 a). Las pérdidas se observan de forma dispersa abarcando toda el área de estudio. La mayor pérdida de especies se produciría entre los 1000 y 2500 msnm y en altitudes mayores a los 4500 msnm (Figura 4 a). También se registran las mayores pérdidas en zonas que están fuera de las dos áreas protegidas, en zonas cubiertas actualmente por: bosque siempreverde montano alto, bosque andino,

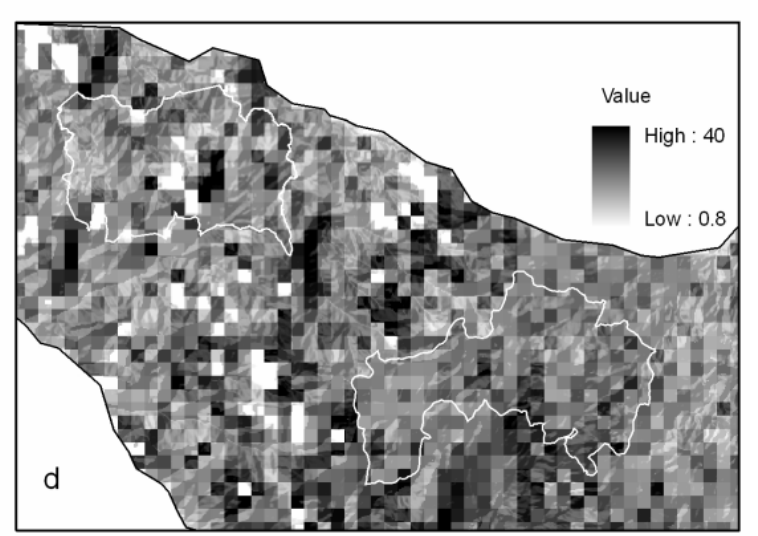

páramo de frailejones, matorral seco, y áreas intervenidas. Al analizar las dos áreas protegidas que son la base del corredor, el modelo muestra que podrían existir pérdida de especies vegetales en un escenario de cambio climático; el Bosque Protector Golondrinas se verá afectado por la pérdida de especies en zonas con bosque andino mientras que la Reserva Ecológica El Ángel en zonas cubiertas por páramo de frailejones, páramo de almohadillas, bosque de Polylepis sp y páramo lacustre (Figura $3 \mathrm{~b}$ ).

Ante el supuesto de dispersión universal o ilimitada, la tasa de recambio temporal de especies se estima que podría variar entre 3.2 - 56\% (Figura $3 \mathrm{c}$ ), con los porcentajes más altos entre $2500-4000 \mathrm{msnm}$

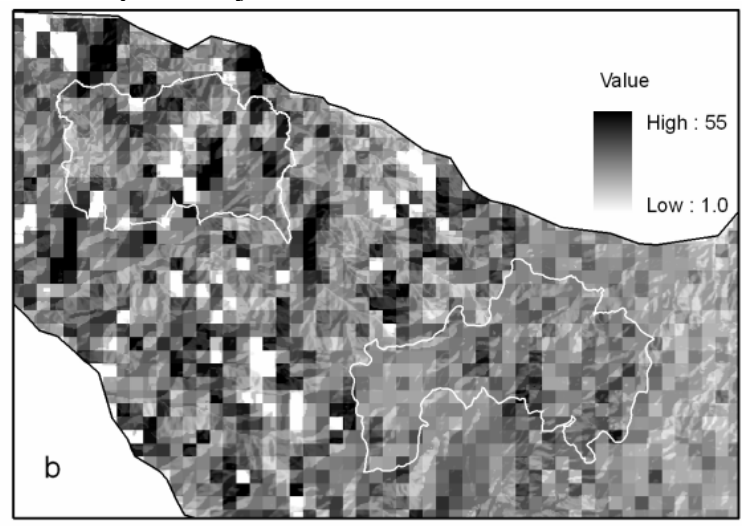

y en altitudes mayores a $4500 \mathrm{msnm}$ (Tabla 1, Figura $4 \mathrm{~b}$ ), en áreas cubiertas por: bosque siempreverde montano alto, bosque andino, páramo de frailejones; encontrándose áreas extensas con alto recambio en zonas fuera de las dos áreas protegidas. En el Bosque Protector Golondrinas podría existir un gran recambio de especies en el bosque andino en el área comprendida entre Chical y Goaltal. En la Reserva Ecológica El Ángel las áreas con mayor intercambio de especies podrían ocurrir en el páramo de almohadillas, páramo de frailejones y zonas actualmente quemadas (Figura $3 \mathrm{c}$ ). 
Taba 1. Valor de la tasa media de recambio por píxel cada $500 \mathrm{~m}$ de altitud (dispersión ilimitada)

\begin{tabular}{crrrrrr}
\hline $\begin{array}{c}\text { Altitud } \\
(\mathrm{msnm})\end{array}$ & $\begin{array}{c}\text { Número } \\
\text { píxeles }\end{array}$ & $\begin{array}{c}\% \\
\text { Mínima }\end{array}$ & $\begin{array}{c}\text { \% } \\
\text { Máxima }\end{array}$ & Rango & Media & $\begin{array}{c}\text { Desv. } \\
\text { Estándar }\end{array}$ \\
\hline $1000-1500$ & 112 & 7.00 & 45.75 & 38.75 & 18.96 & 8.17 \\
$1500-2000$ & 253 & 5.77 & 36.81 & 31.04 & 18.54 & 6.69 \\
$2000-2500$ & 254 & 6.49 & 44.44 & 37.95 & 18.69 & 8.36 \\
$2500-3000$ & 106 & 6.90 & 50.33 & 43.43 & 24.06 & 9.08 \\
$3000-3500$ & 321 & 7.45 & 55.94 & 48.49 & 22.03 & 8.46 \\
$3500-4000$ & 332 & 6.67 & 45.67 & 39.00 & 19.13 & 6.69 \\
$4000-4500$ & 40 & 3.17 & 35.90 & 32.73 & 15.19 & 5.72 \\
$4500-5000$ & 1 & 20.14 & 20.14 & 0.00 & 20.14 & 0.00 \\
\hline
\end{tabular}

Bajo el supuesto de dispersión nula el cambio en composición de especies es menor y podría fluctuar entre 0.8 - 40\% (Figura $3 \mathrm{~d}$ ), las zonas con mayor alteración estarían entre los 2500 - 4000 msnm y en altitudes mayores a los $4500 \mathrm{msnm}$ (Tabla 2, Figura 4 b). Los cambios en la composición florística se producirían en las mismas formaciones vegetales que el supuesto de dispersión ilimitada.

Tabla 2 .Valor de la tasa media de recambio por píxel cada $500 \mathrm{~m}$ de altitud (dispersión nula).

\begin{tabular}{crrrrrr}
\hline $\begin{array}{c}\text { Altitud } \\
(\mathrm{msnm})\end{array}$ & $\begin{array}{c}\text { Número } \\
\text { píxeles }\end{array}$ & $\begin{array}{c}\% \\
\text { Mínimo }\end{array}$ & $\begin{array}{c}\% \\
\text { Máximo }\end{array}$ & Rango & Media & $\begin{array}{c}\text { Desv. } \\
\text { Estándar }\end{array}$ \\
\hline $1000-1500$ & 112 & 1.65 & 29.89 & 28.24 & 9.97 & 5.34 \\
$1500-2000$ & 253 & 2.08 & 25.36 & 23.28 & 10.59 & 4.68 \\
$2000-2500$ & 254 & 1.31 & 36.36 & 35.05 & 10.29 & 5.51 \\
$2500-3000$ & 106 & 3.57 & 39.52 & 35.95 & 13.24 & 6.95 \\
$3000-3500$ & 321 & 1.14 & 37.96 & 36.82 & 12.13 & 6.79 \\
$3500-4000$ & 332 & 2.25 & 36.11 & 33.86 & 11.41 & 5.56 \\
$4000-4500$ & 40 & 0.81 & 24.24 & 23.43 & 9.40 & 4.81 \\
$4500-5000$ & 1 & 12.88 & 12.88 & 0.00 & 12.88 & 0.00 \\
\hline
\end{tabular}

\section{Conclusiones.}

Los resultados de esta investigación ofrecen una primera visión de los posibles impactos del cambio climático en un área rica en biodiversidad y ayudan a comprender sus efectos sobre la misma. Nunca se podrá predecir el futuro con exactitud, pero se necesita una estrategia que permita mejorar la comprensión de los efectos probables del clima futuro sobre la biodiversidad. La información proporcionada debe ser manejada con precaución, teniendo en cuenta sus limitaciones y comprendiendo que son aproximaciones de lo que podría suceder en el 2080. Simulaciones más realistas de los efectos del cambio climático en la distribución de las especies requieren una mejor comprensión de las complejas interacciones entre los muchos factores que afectan la distribución de especies.

La técnica estadística Multivariate Adaptive Regression Splines (MARS) proporciona modelos de distribución potencial fáciles de interpretar. Hay que tener claro que independientemente de la técnica con la que se generen los modelos, sólo representan un primer paso para comprender los impactos potenciales del cambio climático sobre hábitats y especies, ya que toma en cuenta solo el cambio climático y deja de lado las interacciones entre especies, capacidad de migración, disponibilidad de hábitat, etc. Al poseer esta información es posible tener una imagen incompleta de las verdaderas amenazas del cambio climático sobre la biodiversidad.

La riqueza potencial futura del área de estudio varía de acuerdo a los datos empleados para proyectar las posibles distribuciones futuras de las especies. Es decir la riqueza futura de la zona podría experimentar una ligera pérdida bajo el supuesto de dispersión ilimitada, pero podría haber una pérdida importante bajo el supuesto de dispersión nula. Sin embargo, bajo cualquiera de estas dos asunciones extremas de dispersión, se mantiene estable la alta riqueza de la parte occidental del área en estudio.

Aunque las pérdidas son relativamente pocas, la alta tasa de recambio ( $56 \%$ bajo dispersión ilimitada y $40 \%$ bajo dispersión nula) revela que algunos de los principales ecosistemas existentes en el área de estudio (bosque andino, bosque siempreverde montano alto y todos los tipos de páramos) estarían en el futuro fuertemente amenazados. La ganancia y pérdida de especies ocurridas en una misma área con la misma intensidad, provocan un cambio en la composición florística inicial y una consecuente alteración de los ecosistemas existentes en la actualidad. Las zonas con tasas de recambio alto son: parte central y oriental del Bosque Protector Golondrinas, centro y oriente de la Reserva Ecológica El Ángel y los ecosistemas que están conectando estas dos áreas protegidas. El alto recambio esperado para la zona de estudio ofrece nuevos retos al potenciar la aparición de comunidades vegetales nuevas con características funcionales desconocidas.

En áreas que en futuro se estima un riesgo de pérdida de riqueza es necesario recolectar semillas destinadas a los bancos de germoplasma. Para las áreas que mantengan su riqueza estable en el futuro, las personas tomadoras de decisión a nivel nacional y local tendrán que enfocar sus esfuerzos para detener las presiones $\mathrm{y}$ fuentes de presión que puedan degradar la situación actual. En cambio en zonas cuyos valores de biodiversidad actual vayan a verse incrementados en el futuro se deben proponer estrategias de manejo que faciliten este proceso.

El cambio climático es inevitable, pero si se continúa trabajando en conservar los ecosistemas naturales que conectan altitudinalmente el Bosque Protector Golondrinas y la Reserva Ecológica El Ángel, por lo menos se puede asegurar que las 

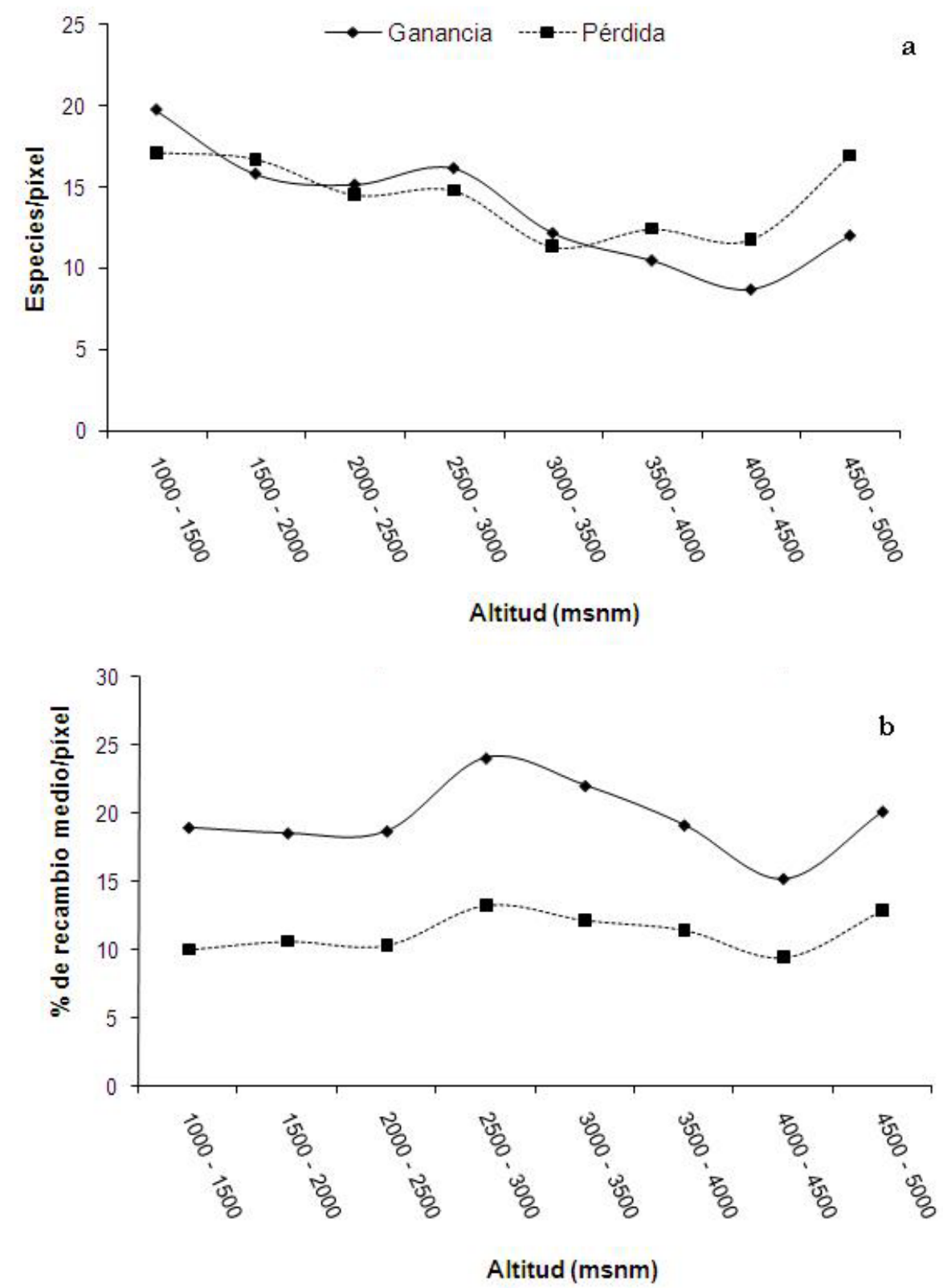

Figura 4. Patrones espaciales de cambio (HadCM3-A2, 2080). a. ganancia y pérdida media de especies por píxel cada $500 \mathrm{~m}$ de altitud, b. Porcentaje de recambio medio (Species turnover), por píxel cada $500 \mathrm{~m}$ de altitud, (dispersión ilimitada y dispersión nula).

especies tendrán espacios naturales a donde migrar y evitar su extinción por falta de hábitats. En base a esta experiencia local, se propone la creación de corredores altitudinales entre áreas naturales protegidas, como una estrategia para adaptación y conservación de los ecosistemas de montaña ante los efectos del cambio climático, asegurando así, la migración y protección de la biodiversidad.

\section{Agradecimientos.}

Esta investigación y las actividades que la respaldaron fueron realizadas gracias al soporte de Proyecto Conservación Comunitaria Fase II financiado por la John D. and Catherine T. MacArthur Foundation y ejecutado por la Corporación Grupo Randi Randi. Agradecemos los comentarios y apoyo de: Telma Paredes, Pablo Cabrera, Carla Gavilanes, César Cisneros, Susan V. Poats, Ángel Felicísimo y Alicia Gómez-Muñoz.

\section{Literatura citada.}

Araújo M.B. \& Williams P.H. 2000. Selecting areas for species persistence using occurrence data. Biological Conservation. 96: 331-345.

Arnell N.W., Livermore M.J.L., Kovats S., Levy P.E., Nicholls R., Parry M.L. \& Gaffin S.R. 2004. Climate and Socio- economic scenarios for global- scale climate 
change impacts assessments: characterising the SRES storylines. Global Environmental Change. 14: 3-20.

Chapin F.S.III., Zavaleta E.S., Eviner V.T., Naylor, R.L., Vitousek P.M., Reynolds H.L., Hooper D.U., Lavorel S., Sala O.E., Hobbie S.E., Mack M.C. \& Díaz S. 2000. Consequences of changing biodiversity. Nature. 405: 234-242.

Cuesta-Camacho F., Ganzenmüller A., Peralvo M.F., Sáenz M., Novoa J. \& Riofrío G. 2006. Predicting specie's niche distribution shifts and biodiversity change within climate change scenarios. A regional assessment for bird and plant species in the Northern Tropical Andes. Nacional Institute for Public Heath and the Environment EcoCiencia/MNP_Andes. 1-36.

Delgado T. 2008. Evolución de la diversidad vegetal en Ecuador ante un escenario de cambio global. Memoria de Tesis Doctoral. Madrid: Departamento de Publicaciones. Universidad Complutense de Madrid. $\mathrm{hptt} /$ /www.kraken.unex.es/kraken

Elith J., Graham C.H., Anderson R.P., Dudík M., Ferrier S., Guisan A., Hijmans R.J., Huettmann F., Leathwick J.R., Lehmann A., Li, J. Lohmann L.G., Loiselle B.A., Manion G., Moritz C., Nakamura M, Nakazawa Y., McC. Overton J., Peterson A.T., Phillips S.J., Richardson K., Scachetti-Pereira R., Schapire R.E., Soberón J., Williams S., Wisz M.S. \& Zimmermann N.E. 2006 Novel methods improve prediction of species' distributions from occurrence data. Ecography. 29: 129-151.

-------. \& Leathwick J. 2007 Predicting species distributions from museum and herbarium records using multiresponse models fitted with multivariate adaptive regression splines. Diversity and Distributions. 13: 265275.

Fielding A.H. \& Bell J.E. 1997. A review of methods for the assessment of prediction errors in conservation presence/absence models. Environmental conservation. 24(1): 38-49.

Friedman J.H. 1991. Multivariate adaptive regression splines. Annals of Statistics. 19: 1-141.

Graham C.H., Ferrier S., Huettman F., Moritz C. \& Peterson A.T. 2004. New developments in museum-based informatics and applications in biodiversity analysis. TRENDS in Ecology and Evolution. 19(9): 497-502.

Guisan A. \& Thuiller W. 2005. Predicting species distribution: offering more than simple habitat models. Ecology Letter. 8: 993-1009.

-----------., Lehmann A., Ferrier S., Austin M., Overton J.M, Aspinall R. \& Hastie T. 2006. Making better biogeographical predictions of species' distribution. Journal of Applied Ecology. 43: 386-392.

Hanley J.A. \& McNeil B.J. 1982. The meaning and use of the area under a Receiver Operating Characteristic (ROC) Curve. Radiology. 143: 29-36.

Hijmans R.J., Cameron S.E., Parra J.L., Jones P.G. \& Jarvis A. 2005. Very high resolution interpolated climate surfaces for global land areas Global Change Biology. International Journal of Climatology. 25: 1965-1978.

Hijmans R.J. \& Graham C.H. 2006. The ability of climate envelope models to predict the effect of climate change on species distributions. Global Change Biology. 12: 2272-2281.

Hulme M. \& Sheard N. 1999. Escenarios de Cambio
Climático para Países de los Andes del Norte. Unidad de Investigación Climática, Norwich, Reino Unido.

IPCC (Grupo Intergubernamental de Expertos sobre Cambio Climático). 2000. Informe especial del IPCC. Escenarios de Emisiones. Resumen para responsables de políticas. Grupo de Trabajo III.

IPCC (International Panel on Climatic Change). 2007 "Climate Change 2007: Impacts, Adaptation and Vulnerability". Sumary for Policymakers Working Group II. Fourth Assessmente Report.

Leathwick J.R., Elith J. \& Hastie T. 2006. Comparative performance of generalized additive models and multivariate adaptive regression splines for statistical modelling of species distributions. Ecological Modelling. 199: 188-196.

Liu C., Berry P.M., Dawson T.P. \& Pearson G.P. 2005. Selecting thresholds of occurrence in the prediction of species distributions. Ecography. 28: 385-393.

Martínez-Meyer E. 2005. Climate change and biodiversity: Some considerations in forescasting shifts in species' potential distributions. Biodiversity Informatics. 2: 4255.

Moisen G.G. \& Frescino T.S. 2002. Comparing five modelling techniques for predicting forest characteristics. Ecological Modelling. 157: 209-225.

Muñoz J. \& Felicísimo A.M. 2004. Comparison of statistical methods commonly used in predictive modelling. Journal of Vegetation Science. 15(2): 285-292.

Myers N., Mittermeier R., Mittermeier C., da Fonseca G. \& Kent J. 2000. Biodiversity hotspots for conservation priorities. Nature. 403: 853-858.

Parmesan C. \& Yohe G. 2003. A globally coherent fingerprint of climate change impacts across natural systems. Nature. 421: 37-42.

Pearson R.G. 2006. Climate change and the migration capacity of species. TRENDS in Ecology and Evolution. 21(3): 111-113.

Peterson A.T., Ortega-Huerta M.A., Bartley J., SánchezCordero V., Soberón J., Buddemeier R.H. \& Stockwell D.R.B. 2002. Future projections for Mexican faunas under global climate change scenarios. Nature. 416: 626-629.

Root T.L., Price J.T., Hall K.R., Schneider S.H., Rosenzweig C. \& Pounds J.A. 2003. Fingerprints of global warming on wild animals and plants. Nature. 421: $57-60$.

Swets J.A. 1988. Measuring accuracy of diagnostic sytems. Science. 240: 1285-1293.

Thomas C.D., Cameron A., Green R.E., Bakkenes M., Beaumont L.J., Collingham Y.C., Erasmus B.F.N., Ferreira de Siqueira M., Grainger A., Hannah L., Hughes L., Huntley B., van Jaarsveld A.S., Midgley G.F., Miless L., Ortega-Huerta M.A., Peterson A.T., Phillips O.L. \& Williams S.E. 2004. Extinction risk from climate change. Nature. 427: 145-148.

Thuiller W. 2004. Patterns and uncertainties of species' range shifts under climate change. Global Change Biology. 10: 2020-2027. --., Lavorel S., Araújo B., Sykes M.T. \& Prentice C.I. 2005a. Climate change threats to plant diversity in Europa. Proceedings of the National Academy of Sciences of the USA, PNAS. 102: 8245-8250.

-----., Richardson D.M., Pysek P., Midgley G.F., Hughes G.O. \& Rouget M. 2005b. Niche-based 
modelling as a tool for predicting the risk of alien plant invasions at a global scale. Global Change Biology. 11: 2234-2250.

-------., Albert C., Araújo M.B., Berry P.M., Cabeza M., Guisan A., Hickler T., Midgley G.F., Peterson J., Schurr F.M., Sykes M.T. \& Zimmermann N.E. 2008. Predicting global change impacts on plant species' distributions: Future challenges. Perspectives in Plant Ecology, Evolution and Systematics. 9: 137-152.

Valencia R., Cerón C., Palacios W. \& Sierra R. 1999.
Formaciones Vegetales de la Sierra del Ecuador. En: Sierra R. (eds) Propuesta Preliminar de un Sistema de Clasificación de Vegetación para el Ecuador Continental: 79-108. Proyecto INEFAN/GEF-BIRF y EcoCiencia. Quito, Ecuador

Walther G.R., Post E., Convery P., Menzel A., Parmesan C., Beebee T.J.C., Fromentin J-C., Hoegh-Guldberg O. \& Bairlein F. 2002. Ecological responses to recent climate change. Nature. 416: 389-395.

1 Herbario Reinaldo Espinoza (LOJA), Casilla 11-01-249. Loja-Ecuador, delgado_tania@hotmail.com.

2 Corporación Grupo Randi Randi, Casilla17-11-6102, Quito-Ecuador, davsua2@yahoo.com 